\title{
Measurements of Effective Quasiparticle Recombination Times and of Densities of Electronic States at the Fermi Level in Superconducting $\mathrm{Al}-$ and $\mathrm{Pb}$-Films
}

\author{
P.W. Epperlein ${ }^{\star}$ and W. Eisenmenger \\ Universität Stuttgart, Physikalisches Institut, Stuttgart, Federal Republic of Germany
}

Received August 21, 1978

Temperature-dependent quasiparticle recombination lifetimes $\tau_{\text {exp }}(T)$ and densities $N_{0}$ of electronic states at the Fermi level have been measured from time decay experiments of excess quasiparticle concentrations in evaporated, superconducting $\mathrm{Al}$ - and $\mathrm{Pb}$-tunnel junctions. Current pulses were used to inject excess, nonthermal quasiparticles in a single junction acting simultaneously as generator and detector. The experimental lifetimes in "unperturbed" A1 show satisfactory agreement with calculations based on the $2 \Delta$-phonon trapping lifetime model. $\tau_{\exp }$ decreases with increasing perturbations of the Al film structure by oxygen background evaporation. In $\mathrm{Pb}$ the measured times indicate $2 \Delta$ phonon volume losses. The densities $N_{0}$ in $\mathrm{Pb}$-films and "unperturbed" as well as oxygen-perturbed Al-films differ by less than $5 \%$ from the corresponding bulk material data. Therefore, in trying to explain the enhancement of the transition temperature from $1.23 \mathrm{~K}$ to $1.85 \mathrm{~K}$ in perturbed, granular Al-films a change of $N_{0}$ can be ruled out.

\section{Introduction}

In a previous publication [1] we reported on directly measured effective quasiparticle recombination lifetimes $\tau_{\text {exp }}$ in superconducting tin tunnel junctions and, for the first time, on a new experimental method to determine the absolute value of the density $N_{0}$ of electronic states at the Fermi level in thin, vacuumdeposited superconductor films. The experimental times showed good agreement with values calculated on the basis of the ray-acoustic lifetime model [2] in the linear thickness range of $\tau_{\exp }$ with negligible $2 \Delta$ phonon loss processes in the film volume. Moreover, $N_{0}$ of polycrystalline Sn-films with only slightly enhanced $(1 \%)$ transition temperatures $T_{c}$ deviated less than $5 \%$ from that evaluated from the experimental electronic heat-capacity coefficient $\gamma$ of ordinary bulk tin. In the present paper experimental $\tau_{\text {exp }}(T)$ and $N_{0}$ data for $\mathrm{Al}$ and $\mathrm{Pb}$ have been determined. A brief description of the experimental technique and of the lifetime model used, together with the most important results, is given in Sects. II.A and II.B. The experimental data are compared with theory and

\footnotetext{
* Present address: IBM Zürich, Research Laboratory, CH-8803
} Rüschlikon, Switzerland discussed with respect to the questions whether the measured increase of $T_{c}$ in perturbed Al-films may be caused by a change of $N_{0}$ and whether $\tau_{\text {exp }}$ is changed with increasing film structure perturbations (see Sects. II.C and III.A). Experiments on lead were made in order to investigate the influence of phonon volume losses on the value of $\tau_{\exp }$ (see Sect. III.B).

\section{Theory. Brief Outlines}

A. Determination of $N_{0}$ and $\tau_{\exp }$

Using the relations for the BCS density $N_{\mathrm{th}}$ of thermally excited quasiparticles, the lifetime $\tau_{\text {exp }}$ from the rate equations for quasiparticle recombination [3] and the excess quasiparticle density $\delta N$ under a stationary injection rate $I_{0}$ we derived in [1] the following expression for $N_{0}$ :

$N_{0}=\frac{2 I_{0} \tau_{\exp , 0} \exp \left[\Delta(T) / k_{B} T\right]}{\left[x^{2}(0)+2 x(0)\right]\left[2 \pi \Delta(T) k_{B} T\right]^{1 / 2}}$. 
Accordingly, a measurement of $N_{0}$ requires absolute values of the temperature $T$, the energy gap $\Delta(T)$, the rate $I_{0}$, the effective lifetime $\tau_{\exp , 0}$ at $\delta N / N_{\text {th }} \ll 1$ and the relative quasiparticle overinjection $\delta N(t=0) / N_{\mathrm{th}}$ $=x(0)$ at the switch-off time $t=0$ of the injection pulse.

These quantities can be measured with a single, symmetrical tunnel junction, i.e. $\Delta$ and $T$ from the current (i)-voltage $(U)$-characteristics and $x(0)$ and $\tau_{\exp , 0}$ from the time decay of $\delta N$ after pulse excitation. The junction is biased in the thermal tunneling current range $\left(0<U_{B}<2 \Delta / e\right)$ and acts as its own detector. In the present paper current pulses have been used to generate $\delta N$. By current injection additional, nonequilibrium quasiparticles are generated in single particle tunneling via breaking up Cooper-pairs $(U \geqq 2 \Delta / e)$ in the tunneling volume $V_{T u}$ with the rate $I_{0}=2 i_{\text {eff }} / e V_{T u} ; i_{\text {eff }}$ is an effective excitation current (see [1]).

Experimentally $\delta N / N_{\text {th }}$ is determined from the corresponding increase of the thermal tunneling current $i_{\text {th }}$, i.e. $\delta N / N_{\mathrm{th}}=\delta i / i_{\mathrm{th}}$, and $\tau_{\exp , 0}$ can directly be observed from the time decay of $\delta i$ sufficiently long after switch-off of the injection pulse. Accurate values for $x(0)$ and $\tau_{\exp , 0}$ are obtained from a mean least squares fit of the nonexponential decay function [1]

$x(t)=2\left\{[1+2 / x(0)] \cdot \exp \left(t / \tau_{\exp , 0}\right)-1\right\}^{-1}$

to the experimental decay curves.

Electronic densities $N_{0}$ determined from (1) for evaporated film samples are compared with values computed from the experimental electronic heat-capacity coefficient $\gamma$ of the bulk material according to the formula

$N_{0}=3 \gamma / \pi^{2} k_{B}^{2}=N_{0}^{\mathrm{bs}}(1+\lambda)$,

where $N_{0}^{\text {bs }}$ is the band-structure density of states and $(1+\lambda)$ the enhancement factor due to the electronphonon coupling $\lambda[4]$.

\section{B. Quasiparticle Lifetime Model}

According to the comprehensive calculations for the effective quasiparticle recombination lifetimes in superconductors [2], the influence of phonon reemission and reabsorption results in three characteristic ranges of the thickness $(d)$ dependence of $\tau_{\exp }$. As to our junctions, range 2 with $d>\Lambda_{w}$ is the most important one, where $\tau_{\exp }$ can be expressed by a linear thickness dependence

$\tau_{\exp }=d \frac{N_{\mathrm{th}}}{N_{\omega, \mathrm{th}}}\left(\frac{1}{c_{t}^{3}}+\frac{1}{2 c_{l}^{3}}\right) /\left(\frac{\bar{T}_{t}}{c_{t}^{2}}+\frac{\bar{T}_{l}}{2 c_{l}^{2}}\right)$.
In (4) $N_{\omega, \text { th }}$ designates the total density of thermally excited $2 \Delta$-phonons, $c_{l, t}$ are the longitudinal and transverse sound velocities respectively, and $\bar{T}_{t, l}$ $=\bar{T}_{t, l ; \mathrm{f} / \mathrm{s}}+\bar{T}_{t, l ; \mathrm{f} / \mathrm{He}}$ is the total, polarization and angle dependent, average phonon transmission factor at the boundaries film (f)/substrate (s) and film/helium.

We compare our experimental times in $\mathrm{Al}$ and $\mathrm{Pb}$ with (4) assuming dominating $2 \Delta$-phonon escape across the junction boundaries (surface losses), i.e. the validity of the relation $\Lambda_{v} \gg \Lambda_{w}$ between the mean free paths of $2 \Delta$-phonons with respect to reabsorption $\left(\Lambda_{w}\right)$ and bulk loss processes $\left(A_{v}\right)$ (volume losses without pairbreaking).

\section{C. $T_{c}$ as a Function of $N_{0}$}

In this subsection we evaluate the exclusive dependence of $T_{c}$ on $N_{0}$, the other parameters as for instance the average squared phonon frequency $\left\langle\omega^{2}\right\rangle$ being constant [5], by using the improved and more general McMillan-Equation for $T_{c}$ [4] of Garland and Allen (see Eq. (14) in [6]). With this calculation we are able to predict quantitatively the $T_{c}$-enhancement in perturbed Al-films caused by an eventual change of $N_{0}$.

The work of Garland and Allen takes account of deviations in the shape of the phonon distribution $F(\omega)$ from the model of niobium used in McMillan's original paper and results in the following $T_{c}$-expression

$T_{c}=(0.25+0.67 x) \omega_{0}$

$\cdot \exp \left[-\frac{1+\lambda}{\left(0.53+0.62 x^{1 / 2}\right) \lambda-\mu^{*}-(0.25+0.67 x) \lambda \mu^{*}}\right]$

with $x=\left\langle\omega^{2}\right\rangle / \omega_{0}^{2}, \omega_{0}$ is the upper cut-off phonon frequency. For $\mathrm{Al}$ we have from available experiments [7] $\left\langle\omega^{2}\right\rangle=849 \mathrm{meV}^{2}, \omega_{0}=40 \mathrm{meV}$ (neglecting the slight high-frequency tail in the electron-phonon spectral function $\left.\alpha^{2}(\omega) F(\omega)\right)$ and thus $x=0.53$. According to McMillan $\lambda$ is the electron-phonon coupling constant as defined by

$\lambda \equiv 2 \int_{0}^{\omega_{0}} \alpha^{2}(\omega) F(\omega) \frac{d \omega}{\omega}=\frac{N_{0}\left\langle I^{2}\right\rangle}{M\left\langle\omega^{2}\right\rangle}$

and $\mu^{*}$ is the Coulomb repulsion pseudopotential

$\mu^{*}=N_{0} V_{c} /\left[1+N_{0} V_{c} \ln \left(E_{F} / \omega_{0}\right)\right]$,

where $\alpha^{2}(\omega)$ is an average of the electron-phonon interaction, $\left\langle I^{2}\right\rangle$ is the squared matrix element of the electron-ion-deformation potential averaged over all 


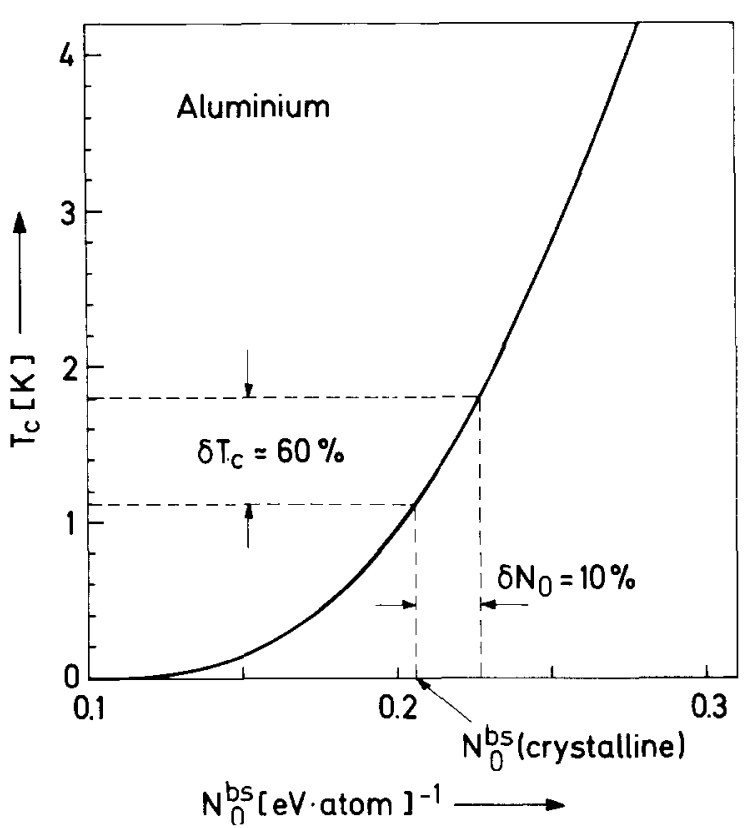

Fig. 1. Variation of the transition temperature $T_{c}$ in aluminium with the band-structure density $N_{0}^{\text {bs }}$ of electronic states at the Fermi level, computed from an improved McMillan-Equation for $T_{\mathrm{c}}$ of Garland and Allen

possible scatterings on the Fermi surface, $M$ is the ionic mass, $E_{F}$ is the Fermi energy and $V_{c}$ is the average over the Fermi surface of the screened Coulomb interaction. $N_{0}$ in (6) and (7) is the bandstructure density of states in the units states $/ \mathrm{eV} \cdot$ atom $\cdot$ spin.

Applying Eq. (5) to $\mathrm{Al}$, where data for $\left\langle I^{2}\right\rangle / M\left\langle\omega^{2}\right\rangle$, $V_{c}$ and $\ln \left(E_{F} / \omega_{0}\right)$ were available [25], we obtained $T_{c}$ as a function of $N_{0}$ as follows

$T_{c}=281 \exp \left[-\frac{1+11.58 N_{0}+17.92 N_{0}^{2}}{0.36 N_{0}+15.97 N_{0}^{2}}\right][\mathrm{K}]$.

Figure 1 shows the graph of (8). From Fig. 1 we take a critical temperature $T_{c}=1.13 \mathrm{~K}$ for crystalline $\mathrm{Al}$ in reasonable agreement with the experimental bulk value of $1.16 \mathrm{~K}$ [4]. Moreover, an increase of $N_{0}$ by $10 \%$ for example causes a $T_{c}$-enhancement by $60 \%$. From band-structure calculations follows [8] that the density of electronic states $N(E)$ in normal Al decreases near the Fermi level with increasing energy, i.e. $\left.\frac{d}{d E} N(E)\right|_{E_{F}}<0$. Hence it follows that a removal of electrons shifts the Fermi level to an energy with a higher density $N_{0}$ (assuming an unchanged shape of $N(E)$ ) and, therefore, results in an enhanced $T_{c}$. Experimentally this effect has been verified $[9,10]$.

\section{Experimental Data and Results. Conclusions}

\section{A. Aluminium}

Since the experiments of Buckel and Hilsch [11] it is well-known that shifted transition temperatures $T_{c}$ in superconductors can be produced by lattice disorder and particle size effects. In these experiments metal films with high disorder were made by evaporation onto substrates held at liquid-helium temperatures. However, by warming up to room temperature the films recrystallized and the critical temperatures approached the unshifted values. These results stimulated all later studies of the structure influence on $T_{c}$.

In our experiments we applied a method developed by Abeles et al. [12] and described in detail in [13]. Perturbed Al-tunnel junctions with granular film structure and with enhanced and stabilized $T_{c}$ were prepared by oxygen background evaporation onto a sapphire substrate held at room temperature. The necessary $\mathrm{O}_{2}$-partial-pressure was maintained either by continuously admitting oxygen into the evaporation system and simultaneously pumping on the evaporator or alternatively by the evaporation of $\mathrm{Al}$ from alumina $\left(\mathrm{Al}_{2} \mathrm{O}_{3}\right)$ coated molybdenum boats (Manufacturers: R.D. Mathis Company). An external $\mathrm{O}_{2}$-supply is not necessary in the last case. We varied the oxygen pressure between $1 \cdot 10^{-4}$ and $5 \cdot 10^{-4}$ Torr (first case). During evaporation the pressure dropped to $2 \ldots 4 \cdot 10^{-5}$ Torr by the getter action of the Al, depending on the evaporation rate which has been varied between 20 and $150 \AA / \mathrm{s}$. By this technique the $T_{c}$ of the Al-junctions was increased to $1.85 \mathrm{~K}$. Samples with higher $T_{c}$ showed high asymptotic resistances $R_{\infty}$ resulting in too high differential resistances $R_{d}$ in the thermal tunneling current region for our electronic measuring method to be applicable (compare Sect. III in [1]). "Unperturbed" samples with a slightly enhanced $T_{c}$ of $1.23 \mathrm{~K}$ were obtained by evaporation with a rate of $150 \AA / \mathrm{s}$ at a residual pressure of $10^{-6}$ Torr.

A special stripline like junction configuration guaranteed a negligibly low inductive crosstalk $(-60 \mathrm{~dB})$ from the high excitation current pulse to the voltage (signal) terminals. The tunnel junction (with an area of $0.3 \mathrm{~mm}^{2}$ ) is shaped between two superconducting films elsewhere insulated by an approximately $3000 \AA$ thick $\mathrm{SiO}$ layer (for more details see Fig. 3 in [1]). The tunnel barrier was formed by room temperature oxidation of the bottom film through the $\mathrm{SiO}$ window at an $\mathrm{O}_{2}$-pressure of 20 to 30 Torr for 3 to $4 \mathrm{~min}$. Samples could be prepared with suitable properties, i.e. $R_{\infty}$ in the $10 \mathrm{~m} \Omega$-range, $R_{d}$ in the $1 \Omega$-range and ratios $i_{2 \Lambda_{+}} / i_{2 \Lambda_{-}}$between the maximal and minimal 


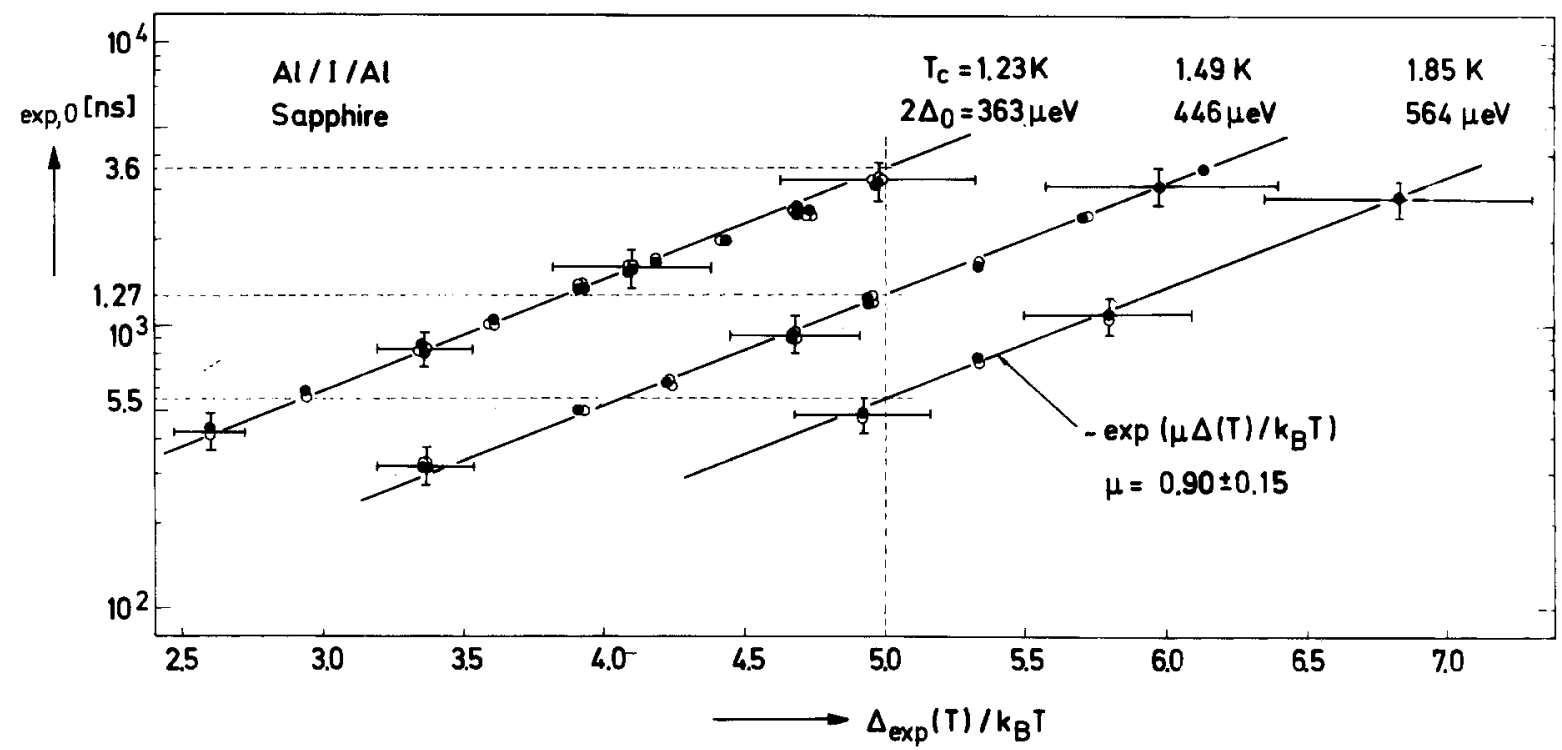

Fig. 2. Experimental quasiparticle recombination lifetimes versus $\Delta / k_{B} T$ for three Al-tunnel junctions with different degrees of perturbation and direct contact to liquid ${ }^{3} \mathrm{He}$. Symbols $(\bullet)$ and $(0)$ correspond to lifetimes obtained from the slopes of the logarithmic diagrams of the decay curves at long times and from the fits of the nonexponential decay function (2) on the experimental decay curves, respectively

single particle current at $U=2 \Delta / e$ of greater than 150 ; this ratio is a measure for the junction quality. The specimens were directly immersed in liquid ${ }^{3} \mathrm{He}$ with a lowest attainable temperature of $0.38 \mathrm{~K}$. The bath temperature was measured with a calibrated germanium resistance and by the vapor-pressure (determined by a Baratron pressure meter, MKS Instruments). Additionally, we also checked these values by determining the temperature from the thermal tunnel currents of the junctions; (deviations $\pm 0.01 \mathrm{~K}$ ).

The time constants $\tau_{\exp , 0}$ directly measured from the time decays of the pulse signals at low excess quasiparticle densities are plotted as a function of $\Delta(T) / k_{B} T$ for three Al-tunnel junctions with different $T_{c}$ values in Fig. 2. Within the experimental errors the $\tau_{\text {exp }, 0}$ 's verify the exponential $\exp \left(\Delta(T) / k_{B} T\right)$ temperature dependence required by theory, i.e. the times are proportional to the reciprocal concentration of thermally excited quasiparticles. Hence it follows, that these time constants can be ascribed to the effective quasiparticle recombination lifetimes in Al-tunnel junctions.

We now compare our experimental value $\tau_{\exp , o} / d$ $=0.024( \pm 20 \%)[\mathrm{s} / \mathrm{cm}]$ for the decay time constant in the "unperturbed" Al-junction $(d=5000 \AA, \Delta=$ $178 \mu \mathrm{eV}, T_{c}=1.23 \mathrm{~K}$ ) at $\Delta=4 k_{B} T$ and $\Delta=200 \mu \mathrm{eV}$ with the calculation according to (4) and with measurements of Long [14]. The decay time was corrected to the energy gap of $200 \mu \mathrm{eV}$ for the same $\Delta / k_{B} T$ by the relation $\tau_{\exp .0} \propto d \cdot \Delta^{-2} \cdot f\left(\Delta / k_{B} T\right)[2]$, [14] resulting from Eq. (4). This relation has been verified by experiments $[14] ; f$ is a function of the variable $\Delta / k_{B} T$.

Using the acoustic data for $\mathrm{Al}$ on sapphire substrates [2], the phonon transmission coefficient into ${ }^{3} \mathrm{He}$ $\bar{T}_{\mathrm{A} / / 3} \mathrm{He} \approx 0.3[19]$ and the experimental value for $N_{0}$ listed in Table 2, the calculation from (4) valid for $d>\Lambda_{w}\left(\Lambda_{w}=3500 \AA\right.$ experimentally obtained by Long [14] for transverse phonons) and $\Lambda_{v} \gg \Lambda_{w}$, i.e. without phonon volume losses, results in $\tau_{\text {exp, } 0} / d=0.03$ $( \pm 20 \%)[\mathrm{s} / \mathrm{cm}]$. The relatively good agreement between measurement and theory shows that $2 \Delta$-phonon scattering at grain boundaries or at the oxide of the tunnel barrier is of low importance in evaporated, "unperturbed" Al-junctions.

Long [14] obtained a mean lifetime $\tau_{\text {exp }, 0} / d=0.088$ [s/cm] at $\Delta=4 k_{B} T$ and $\Delta=200 \mu \mathrm{eV}$ from several steady-state "vacuum measurements" on Al-samples with a sandwichstructure of two tunnel junctions in the thickness range $1000 \AA \leqq d \leqq 3000 \AA$. This time value, however, relates to the "lifetime" of one quasiparticle. For comparison with our "decay time constant" of an excess quasiparticle density Long's "lifetime" value must be divided by a factor two, since the recombination process takes place between two quasiparticles. The indirect determination of $\tau_{\exp }$ by the steady-state method requires the absolute value of the electronic density of states at the Fermi level, for which Long has taken the bare band-structure value $N_{0}^{\text {bs }}=2.43 \cdot 10^{22} \mathrm{eV}^{-1} \mathrm{~cm}^{-3}$ (both spins) instead of the correct quantity $N_{0}^{\gamma}=N_{0}^{\text {bs }} \cdot(1+\lambda)$ [see Table 2] renormalized by the electron-phonon in- 
Table 1. Correction of the apparent lifetime reduction in the perturbed Al-junctions in respect of the different values of thickness $d$ and gap energy $\Delta$. The effective quasiparticle recombination lifetimes $\tau_{\exp , 0}$ of the perturbed Al-junctions are normalized to the values $\Delta_{u}=0.181 \mathrm{meV}, d_{u}=5000 \AA, \tau_{\text {exp }, 0}^{u}=3700 \mathrm{~ns}$ of the "unperturbed" junction (with $T_{c}=1.23 \mathrm{~K}$ ) at $\Delta=5 k_{B} T$ with the relation $\tau_{\exp , 0} \sim d / \Delta^{2}$

\begin{tabular}{lcc}
\hline$T_{c}[\mathrm{~K}]$ & 1.49 & 1.85 \\
$\Delta[\mathrm{meV}]$ & 0.221 & 0.280 \\
$d[\AA]$ & 2,200 & 3,000 \\
$\tau_{\text {exp }, 0}[\mathrm{~ns}]$ at $\Delta=5 k_{B} T$ & 1,270 & 550 \\
$\frac{\tau_{\text {exp }, 0}}{\tau_{\text {exp }, 0}^{u}}$ [experimental] & 0.34 & 0.15 \\
$\frac{\tau_{\text {exp }, 0}^{u}}{\tau_{\text {exp }, 0}^{u}}=\frac{\Delta_{u}^{2} d}{\Delta^{2} d^{u}}$ [calculated] & 0.30 & 0.25 \\
\hline
\end{tabular}

teraction 2. Moreover, the transmission of longitudinal phonons across the junction boundaries has been neglected. Taking account of these arguments, we converted Long's "vacuum lifetime" with Eq. (4) into the value with liquid He-contact and obtained $\tau_{\exp , 0} / d=0.024[\mathrm{~s} / \mathrm{cm}]$ in good agreement with our decay time constant measured at $\Delta=4 k_{B} T$ and $\Delta=200 \mu \mathrm{eV}$.

Figure 2 shows that $\tau_{\text {exp }, 0}$ decreases with increasing $T_{c}$ at a given $A / k_{B} T$. However, for a correct comparison of the decay times in the three samples we have to take into account that the samples have different thicknesses $d$ and gap values $\Delta$. Therefore, the experimental times in the perturbed samples were related to the decay time in the "unperturbed" junction, according to the general relation $\tau_{\exp , 0} \propto d / \Delta^{2} \cdot f\left(\Delta / k_{B} T\right)$ [2], [14]. As can be seen from the data in Table 1, the apparent lifetime reduction in the moderately perturbed sample with a $T_{c}=1.49 \mathrm{~K}$ can be completely described by the $\tau_{\exp , 0}$ relation within the total experimental uncertainty of $15 \%$ (errors in $\tau(5-$ $10 \%), d(5-10 \%), \Delta(3 \%))$. This is in contrast to the excessive $\tau$-reduction of the strongly perturbed junction with $T_{c}=1.85 \mathrm{~K}$. In the validity range of the $\tau_{\text {exp }, 0}$ relation $\left(A_{w}<d ; A_{w}\right.$ phonon reabsorption mean free path, $d=$ junction thickness; no phonon losses in the bulk material) changes of the electron-phonon coupling parameter $\lambda$ enter only by the gap value $\Delta$. Under this condition the excessive $\tau$-reduction can be only explained by an enhanced $2 \Delta$-phonon escape if $N_{0}$ and the sound velocity are assumed to be unchanged. The enhanced 24 -phonon escape is possible by diffuse phonon scattering at the junction boundaries with films of a granular, irregular structure, since diffuse boundary scattering of phonons results in an effective increase of the limiting angle for total reflection. In thinner films $d<A_{w}$ or with bulk phonon losses an increase of the electron-phonon coupling can directly result in an excessively reduced $\tau$. In discussing the enhancement of $\alpha^{2}(\omega) F(\omega)$ in granular aluminium [7] the sound velocity agrees with that of the bulk material at low frequencies $[15$, 16]. Consequently, an essentially unchanged phonon spectrum $F(\omega)$ results within the limits of a Debye model and thus an increase of the squared matrix element $\alpha^{2}(\omega)$ of the electron-phonon interaction should be responsible for the enhancement of the low-frequency tail in $\alpha^{2}(\omega) F(\omega)$. This is in accordance with a reduction of the intrinsic quasiparticle recombination lifetime and also with the $2 \Delta_{0} / k_{B} T_{c}$ data given in Table 2. The ratio $2 \Delta_{0} / k_{B} T_{c}$ being a measure for the electron-phonon coupling increases with increasing perturbations of the Al-film structure. In Table 2 we have listed the mean values of $N_{0}$ (with its plus-minus standard deviation) for the three samples determined from Eq. (1) with experimental data as described in Sect. IIA. The average was calculated in each case from at least ten $N_{0}$-values obtained from measurements at different temperatures. The maximum scatter of the single $N_{0}$-values relating to the mean value amounts to $10 \%$. From (1) we estimated a maximal error for $N_{0}$ of $30 \%$ to $40 \%$ [1]. As Table 2 shows there is no essential change of $N_{0}$ within the investigated transition temperature range $1.23 \mathrm{~K} \leqq T_{c} \leqq 1.85 \mathrm{~K}$ and $N_{0}$ in the oxygen-perturbed, granular Al-films agrees with the bulk value calculated from (3) with the experimental electronic heatcapacity coefficient $\gamma[17,18]$. We compare this

Table 2. Some characteristic parameters of the Al-films, "unperturbed" $\left(T_{c}=1.23 \mathrm{~K}\right)$ and with increasing perturbation by evaporation in an oxygen atmosphere. $\rho_{4.2}=$ resistivity measured at $T=4.2 \mathrm{~K}, l=$ electron mean free path due to elastic scattering, $R=$ film resistance, $d=$ film thickness, $\bar{D}=$ average grain size, determined by electron microscopy

\begin{tabular}{|c|c|c|c|c|c|c|c|c|c|}
\hline $\begin{array}{l}A_{0} \\
{[\mu \mathrm{eV}]}\end{array}$ & $\begin{array}{l}T_{c} \\
{[\mathrm{~K}]}\end{array}$ & $2 \Delta_{0} / k_{B} T_{c}$ & $\begin{array}{l}\delta T_{c} \\
{[\mathrm{~K}]}\end{array}$ & $\begin{array}{l}d \\
{[\AA]}\end{array}$ & $\begin{array}{l}\rho_{4.2} \\
{\left[10^{-6} \Omega \mathrm{cm}\right]}\end{array}$ & $\begin{array}{l}l_{4,2}^{2} \\
{\left[\AA^{2}\right]}\end{array}$ & $\begin{array}{l}\bar{D} \\
{[\AA]}\end{array}$ & $R_{4,2} / R_{300}$ & $\begin{array}{l}\bar{N}_{0}^{\uparrow \downarrow} \\
{\left[10^{22} \mathrm{eV}^{-1} \mathrm{~cm}^{-3}\right]}\end{array}$ \\
\hline 182 & 1.23 & 3.42 & 0.01 & 2,500 & 0.40 & 2,600 & 2,600 & 0.14 & $3.3 \pm 0.05$ \\
\hline 223 & 1.49 & 3.47 & 0.5 & 850 & 5.1 & 205 & 250 & 0.67 & $3.3+0.08$ \\
\hline 282 & 1.85 & 3.54 & 0.6 & 1,600 & 13 & 80 & 130 & 0.84 & $3.4 \pm 0.02$ \\
\hline
\end{tabular}

For comparison: bulk value $N_{0}^{\dagger \downarrow}=3.45 \cdot 10^{22} \mathrm{eV}^{-1} \mathrm{~cm}^{-3}$ from the experimental electronic heat-capacity coefficient. 
result with results from low temperature oxidation experiments performed on thin superconducting metal films [10]. These measurements showed an enhancement of $T_{c}$ in thin $(100 \AA)$ Al-layers. According to $[10]$ the shift of the transition to higher temperatures can be explained by the relative decrease of the electron density in the metal film. This has been explained by the tunneling of electrons from the metal to acceptor levels of oxygen molecules, chemically bound at the outer boundary of the oxide layer. Concerning our $\mathrm{O}_{2}$-perturbed Al-films (prepared at room temperature), however, we have to assume that the oxygen exists in a stoechiometrically bound form $\left(\mathrm{Al}_{2} \mathrm{O}_{3}\right)$ and hence it cannot lead to a stable terminal state of the system metal/oxide layer/adsorbed oxygen being the precondition for the above explanation.

The unchanged, absolute values of $N_{0}$ for the density of electronic states at the Fermi level (Table 2) show, that $N_{0}$ is not important in the enhancement of $T_{c}$ in our granular Al-films. Therefore, other mechanisms, such as alteration of the phonon spectrum or of the electron-phonon interaction may be significant.

\section{B. Lead}

Although the first experimental [20] and theoretical studies $[21,22]$ to determine quasiparticle recombination lifetimes in superconductors have been performed on the strong coupling lead, there were only a few further measurements in the following period [23-26]. The reasons for that are mainly due to experimental problems resulting from the preparation of proper lead tunnel junctions and from the measurement of the relatively short effective lifetimes. In addition, a perfect determination of $\tau_{\exp , 0}$ is complicated by the quasiparticle overinjection $\delta N / N_{\text {th }}$, easily reached in lead for temperatures $T<2 \mathrm{~K}$. In $[20$, $23,24] \tau_{\text {exp }, 0}$ 's are reported, which were indirectly obtained from steady-state measurements on highohmic ( $\Omega$ - to $\mathrm{k} \Omega$-range), thick tunnel junctions in direct contact to liquid He. We report here first temperature-dependent decay time measurements on low-ohmic $(\mathrm{m} \Omega)$, high-quality $\left(i_{2 \Delta_{+}} / i_{2 \Delta_{-}}>200\right) \mathrm{Pb}-$ tunnel junctions under vacuum conditions.

In the preparation of the $\mathrm{Pb}$-junctions we had to dispense with the sample configuration of low inductive crosstalk, used in the Al-experiments and reported in $[1,25]$, since nearly all junctions prepared with an intermediate layer ( $\mathrm{SiO}$ window) showed too high leakage currents. Proper junctions were obtained, however, with the configuration shown in the inset of Fig. 3; the overlap area of both the films amounts to $0.6 \mathrm{~mm}^{2}$. Pulse experiments made on this film configuration resulted in decay

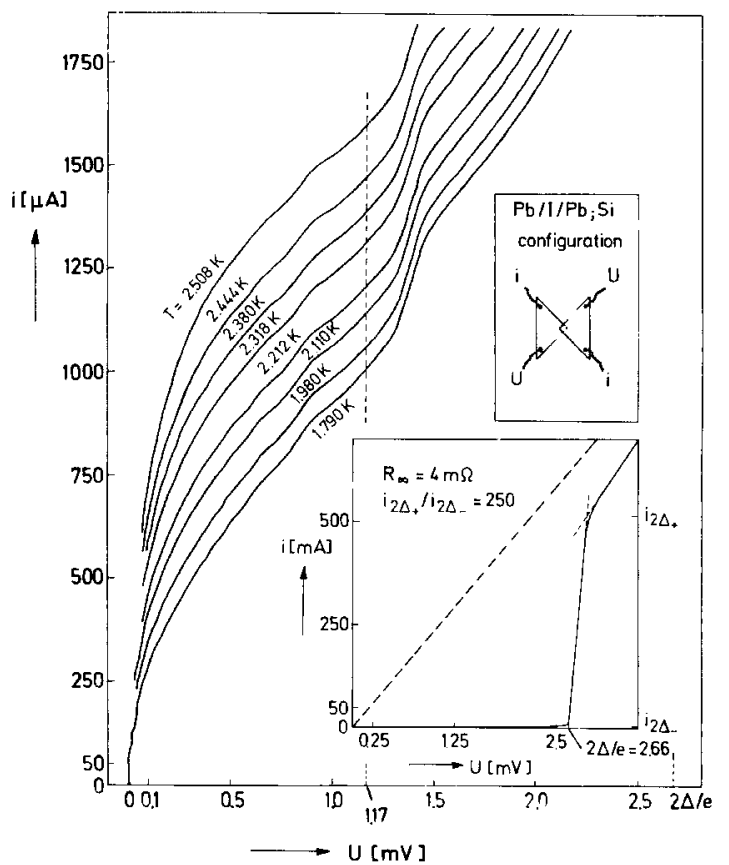

Fig. 3. Current-voltage characteristics of a low-ohmic Pb-tunnel junction for $U<2 \Delta / e$ and $U \lesssim 3 \Delta / e$ (inset). Junction configuration (inset)

curves with slightly superposed crosstalk-induced oscillations.

The lead films were vacuum-deposited onto silicon substrates $(300 \mathrm{~K})$ under a residual gas pressure of $5 \cdot 10^{-7}$ Torr (additional cryopumping by a cold-area $(77 \mathrm{~K})$ around the evaporation source [26]). After the fast evaporation of the bottom film with a rate of $1000 \AA / \mathrm{s}$, the oxidation was immediately started by slowly bleeding ( $20 \mathrm{Torr} / \mathrm{min})$ pure and dry oxygen into the evaporator up to atmospheric pressure. During the oxidation process, taking $1.5 \mathrm{~h}$, the temperature of the substrate and of the vacuum chamber were carefully controlled at $323 \mathrm{~K}$. The first $200 \AA$ of the upper film were slowly deposited with 0.5 to $1 \AA / \mathrm{s}$, then with $200 \AA / \mathrm{s}$. By using this preparation procedure we succeeded in reproducing $\mathrm{Pb}$ junctions with high quality (maximum $i_{2 \Lambda_{+}} / i_{2 \Lambda_{-}} \simeq 10^{4}$ at $T=1.8 \mathrm{~K}$ for a sample with $R_{\infty}=52 \mathrm{~m} \Omega$ ). Figure 3 shows some $i-U$-characteristics in the thermal tunneling range at various temperatures and the characteristic at voltages exceeding $24 / e$ (see inset) of a sample used for the lifetime experiments reported in this paper.

In Figure 4 the experimental time constants $\tau_{\exp , 0}$, directly obtained from the time decays of the pulse signals at $\delta N / N_{\mathrm{th}} \ll 1$, are shown in a logarithmic diagram versus $\Delta / k_{B} T$. The decay time constants verify the exponential temperature law above $T=1.8 \mathrm{~K}$; the reduction of $\tau_{\exp , 0}$ at low temperatures may be due to slightly trapped magnetic flux (junction was 


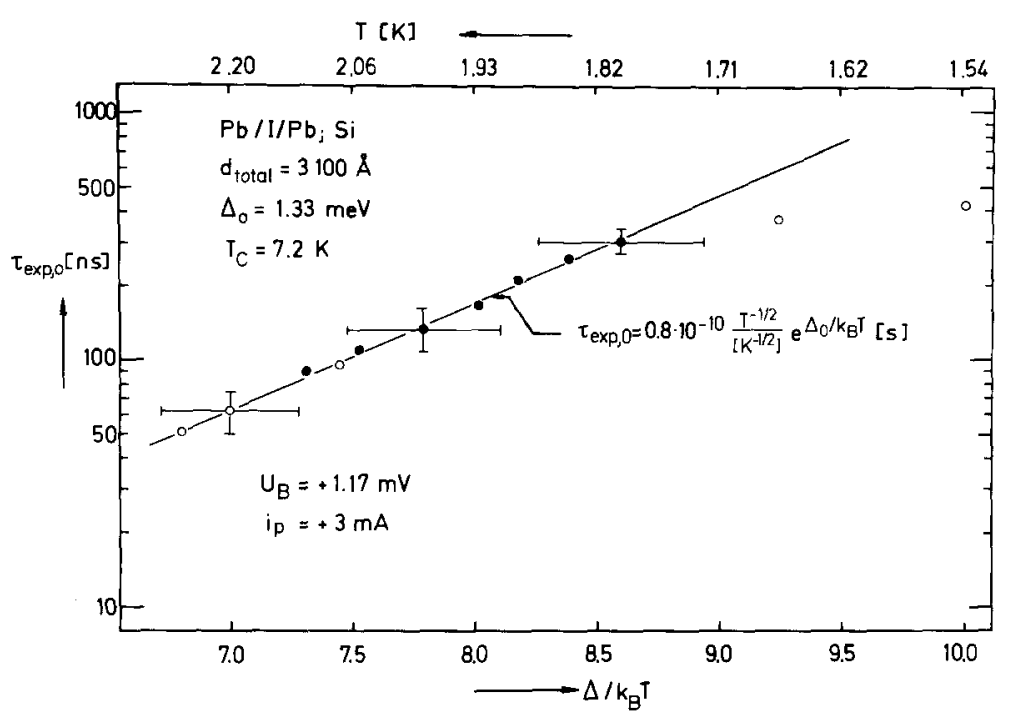

Fig. 4. Experimental quasiparticle recombination lifetimes versus $\Delta / k_{B} T$ for a $\mathrm{Pb}$-tunnel junction under vacuum conditions. Quasiparticle injection by fast current pulses oriented parallel to the geomagnetic field, compare [1]). In order to determine the decay curves the samples were loaded with an average pulse power of $1.5 \cdot 10^{-8} \mathrm{~W}$ with a duty cycle $1: 300$. For checking eventually existing heating effects in our vacuum experiments, the pulse power was reduced to $1.5 \cdot 10^{-10} \mathrm{~W}$ with a duty cycle $1: 30000$. Within the experimental error the decay curves were not changed and the time constants agreed with those in Fig. 4. The experimental temperature dependence of $\tau_{\exp , 0}$, measured in the $3100 \AA$ thick superconducting $\mathrm{Pb}$-tunnel junction under vacuum conditions, can be expressed by

$\tau_{\exp , 0}=\tau_{0} \frac{T^{-1 / 2}}{\left[\mathrm{~K}^{-1 / 2}\right]} \exp \left(\Delta_{0} / k_{B} T\right)$

with $\tau_{0}=0.8 \cdot 10^{-10} \mathrm{~s}$.

This result is in excellent agreement with phonon pulse measurements [26] using $\mathrm{Pb}$-tunnel junctions on silicon as phonon detectors. These measurements [26] showed that the decay time constants $\tau_{\text {exp, }, 0}$ determined in $\mathrm{Pb}$ samples with direct He-contact are only lower by approximately $10 \%$ with respect to the "vacuum-values", i.e. the phonon escape across the junction boundary lead/liquid helium is not the dominant phonon loss process in superconducting $\mathrm{Pb}$.
Moreover, there is no essential dependence of $\tau_{\exp }$ on the junction thickness $d$ in the range $3000 \AA \lesssim d \lesssim 5000 \AA$ [26]. Also indirect steady-state measurements [24] on $3500 \AA$ thick junctions on glass substrates immersed in superfluid He resulted in similar $\tau_{0}$-values, i.e. $\tau_{0}=1.03 \cdot 10^{-10} \mathrm{~s}$ ("lifetime-value" from [24] was multiplied with a factor 0.5 for comparison with our "decay-time constants").

Our experimental "vacuum value" $\tau_{0}=0.8 \cdot 10^{-10} \mathrm{~s}$ is compared with the calculation from Eq. (4) assuming $d>\Lambda_{w}$ and negligible phonon volume losses, i.e. $\Lambda_{v} \gg \Lambda_{w}$. The condition $d>\Lambda_{w}$ is verified by our samples $\left(\Lambda_{w} \simeq 300 \AA[2]\right)$. Using the acoustic data for $\mathrm{Pb}$ on $\mathrm{Si}$ [2] and the experimental value for $N_{0}$ (compare Table 3 ) the calculation results in $\tau_{0}$ $=13.5 \cdot 10^{-10} \mathrm{~s}$ for the $3100 \AA$ thick junction.

The calculated value $\tau_{0}$ is approximately a factor of 17 larger than the experimental result. This difference as well as the good agreement among the above mentioned, different measurements definitely indicate, in contrast to $[24,27,28]$, that 24 -phonon volume loss processes in lead (inelastic phonon scattering at defects and quasiparticles and three-phonon processes) dominate the phonon escape from the sample to the adjoining media substrate and liquid helium.

Finally, we have listed in Table 3 some relevant physical properties of the investigated $\mathrm{Pb}$-films. As in

Table 3. Some characteristic parameters of the lead films, evaporated with high rates of $1000 \AA / \mathrm{s}$ at a residual pressure of $5 \cdot 10^{-7}$ Torr. $\xi=$ coherence length

\begin{tabular}{|c|c|c|c|c|c|c|c|c|c|}
\hline $\begin{array}{l}\Delta_{0}[\mathrm{meV}] \\
\text { at } i_{2{ }_{-}}\end{array}$ & $\begin{array}{l}T_{c} \\
{[\mathrm{~K}]}\end{array}$ & $2 \Delta_{0} / k_{B} T_{c}$ & $\begin{array}{l}\delta T_{c} \\
{[\mathrm{~K}]}\end{array}$ & $\begin{array}{l}d \\
{[\AA]} \\
\end{array}$ & $\begin{array}{l}\rho_{7.4} \\
{\left[10^{-6} \Omega \mathrm{cm}\right]}\end{array}$ & $\begin{array}{l}l_{7} \\
{[\dot{A}]} \\
\end{array}$ & $\begin{array}{l}\xi \\
{[\AA]} \\
\end{array}$ & $R_{7.4} / R_{300}$ & $\begin{array}{l}\bar{N}_{0}^{\uparrow \downarrow} \\
{\left[10^{22} \mathrm{eV}^{-1} \mathrm{~cm}^{-3}\right]}\end{array}$ \\
\hline 1.33 & 7.2 & 4.29 & 0.03 & 2,500 & 0.21 & $>2,500$ & $\begin{array}{l}500 \\
\text { at } 2 \mathrm{~K}\end{array}$ & 0.01 & $4.2 \pm 0.2$ \\
\hline
\end{tabular}

For comparison: bulk value $N_{0}^{\uparrow \downarrow}=4.4 \cdot 10^{22} \mathrm{eV}^{-1} \mathrm{~cm}^{-3}$ from specific-heat measurements. 
tin [1] and aluminium [see Table 2] the density of electronic states $N_{0}$ (with both spins) in evaporated lead films of polycrystalline structure agree with the corresponding "bulk value".

Helpful discussions with K. Laßmann, R. Rolcke, M. Welte and H. Sixl are gratefully acknowledged. We should like to thank Mrs. G. Mayerhöffer for preparing the substrates and Mrs. S. Sauer for the electron microscopy.

\section{References}

1. Epperlein, P.W., Lassmann, K., Eisenmenger, W.: Z. Physik B 31, 377 (1978)

2. Eisenmenger, W., Lassmann, K., Trumpp, H.J., Krauss, R.: Appl. Phys. 11, 307 (1976) and Appl. Phys. 12, 163 (1977)

3. Rothwarf, A., Taylor, B.N.: Phys. Rev. Lett. 19, 27 (1967)

4. McMillan, W.L.: Phys. Rev. 167, 331 (1968)

5. In reality the density of electronic states at the Fermi level, the electron-ion interaction and the phonon frequencies depend on each other in a complex manner (compare [4])

6. Dynes, R.C.: Phys. Rev. B 2, 644 (1970)

7. Leger, A., Klein, J.: Phys. Letters 28 A, 751 (1969)

8. Fritsche, L., Ries, G., Schlïter, M.; DPG, Sommerschule für Supraleitung, Steibis/Allgäu 1969, Page 1

According to Garland, J.W., Bennemann, K.H., Mueller, F.M.: Phys. Rev. Letters 21, 1315 (1968) the smearing of structure in $N(E)$ is relatively small in granular films

9. Glover III, R.E., Sherill, M.D.: Phys. Rev. Letters 5, 248 (1960)

10. Rühl, W.: Z. Physik 186, 190 (1965) and Z. Physik 196, 464 (1966)

11. Buckel, W., Hilsch, R.: Z. Physik 138, 109 (1954)

12. Abeles, B., Cohen, R.W., Cullen, G.W.: Phys. Rev, Letters 17, $632(1966)$

13. Cohen, R.W., Abeles, B.: Phys. Rev. 168, 444 (1968)

14. Long, A.R.: J. Phys. F: Metal Phys. 3, 2040 (1973)
15. Kinder, H.: J. Physique 33, Suppl. C-4, 21 (1972)

16. Forkel, W., Welte, M., Eisenmenger, W.: Phys. Rev. Letters 31, 215 (1973)

17. Kittel, C.: Introduction to Solid State Physics, IIIrd Edition, New York: J. Wiley \& Sons 1967

18. Gopal, E.S.R.: Specific Heats at Low Temperatures, Heywood Books, Eds. K. Mendelssohn, and K.D. Timmerhaus, Page 63, London: Plenum Press 1966

19. We have used this value with reference to the $\bar{T}$-value for ${ }^{4} \mathrm{He}$, as there is no strong difference between the phonon transmission into ${ }^{3} \mathrm{He}$ and ${ }^{4} \mathrm{He}$ at the measured phonon frequencies $\$ 50 \mathrm{GHz}$; compare Challis, L.J., J. Phys. C: Solid State Phys. 7, 481 (1974)

20. Ginsberg, D.M.: Phys. Rev. Letters 8, 204 (1962)

21. Schrieffer, J.R., Ginsberg, D.M.: Phys. Rev. Letters, 5, 207 (1962),

22. Rothwarf, A., Cohen, M.: Phys. Rev. 130, 1401 (1963)

23. Parker, W.H., Williams, W.D.: Phys. Rev. Letters, 29, 924 (1972)

24. Jaworski, F., Parker, W.H., Kaplan, S.B.: Phys. Rev. B 14, 4209 (1976)

25. Epperlein, P.W.: Thesis, Universität Stuttgart (1977)

26. Rolcke, R.: Thesis, Universität Stuttgart (1978)

27. Schuller, I., Gray, K.E.: Phys. Rev. 12, 2629 (1975)

The results from these experiments [27] have been corrected in a subsequent Paper [28] by using the correct value $N_{0}^{\gamma}=N_{0}^{\text {bs }}$ $(1+\lambda)$ for the electronic density of states, as resulting for example from our measurements. Especially, the inelastic phonon processes in lead, suggested in [27] were canceled [28]

28. Schuller, I., Gray, K.E.: Phys. Rev. B 14, 4213 (1976)

P.W. Epperlein

W. Eisenmenger

Physikalisches Institut

Universität Stuttgart

Pfaffenwaldring 57

D-7000 Stuttgart 80 (Vaihingen)

Federal Republic of Germany 